\title{
Matrix Approach to Frobenius-Euler Polynomials
}

\author{
G. Tomaz ${ }^{1,2}$ and H. R. Malonek ${ }^{2}$ \\ 1 Instituto Politécnico da Guarda, 6300-559 Guarda, Portugal \\ gtomaz@ipg.pt \\ 2 Centro de Investigação e Desenvolvimento em Matemática e Aplicações, * \\ Universidade de Aveiro, 3810-193 Aveiro, Portugal \\ hrmalon@ua.pt
}

\begin{abstract}
In the last two years Frobenius-Euler polynomials have gained renewed interest and were studied by several authors. This paper presents a novel approach to these polynomials by treating them as Appell polynomials. This allows to apply an elementary matrix representation based on a nilpotent creation matrix for proving some of the main properties of Frobenius-Euler polynomials in a straightforward way.
\end{abstract}

Keywords: Appell polynomials, Frobenius-Euler polynomials, matrix representation

\section{Introduction}

Frobenius-Euler polynomials are extensions of the well known Euler polynomials. They also belong to the class of Appell sequences [3]. As it is well known, Appell polynomials have remarkable applications in several areas of mathematics, in physics, in chemistry and, in general, in engineering.

Frobenius-Euler polynomials have been discussed by Frobenius ([14]) in the context of number theory and divisibility properties by using their link with Stirling numbers of the second kind. Very recently, the interest on them reemerged as can be seen in the papers [4], [11], [17-19] published in 2012 and 2013 but, surprisingly, besides partially in [18], no use of their Appell polynomial properties have been made.

The aim of this paper is to combine the fact that Frobenius-Euler polynomials belong to the Appell class with the possibility to construct their matrix representation based on the creation matrix defined by

$$
(H)_{i j}= \begin{cases}i, & i=j+1 \\ 0, & \text { otherwise }, \quad i, j=0,1, \ldots, m\end{cases}
$$

\footnotetext{
* This work was supported by Portuguese funds through the CIDMA - Center for Research and Development in Mathematics and Applications, and the Portuguese Foundation for Science and Technology("FCT - Fundação para a Ciência e a Tecnologia"), within project PEst-OE/MAT/UI4106/2014.
} 
The simplicity of the structure of the creation matrix and, particularly, its relation to matrices of Pascal type show new insights in Frobenius-Euler polynomials. Moreover, the matrix approach allows to simplify the proof of several of their main properties.

The outline of the paper is as follows. In Sect. 2 the fundamentals of Appell polynomials and their matrix representation are presented. Sect. 3 is devoted to basic concepts of Frobenius-Euler polynomials and the corresponding matrix representation. In Sect. 4 some identities of Frobenius-Euler polynomials in matrix form are derived as well as a relationship of those polynomials and generalized Apostol-Euler polynomials is established. Finally, some conclusions are presented.

\section{Preliminaries}

Polynomial sequences $\left\{p_{n}(x)\right\}_{n \geq 0}$ whose elements verify the conditions

$$
\frac{d}{d x} p_{n}(x)=n p_{n-1}(x), \quad n=1,2, \ldots,
$$

and

$$
p_{0}(x)=c_{0}, \quad c_{0} \in \mathbb{R} \backslash\{0\}
$$

are called Appell sequences (cf. [3]).

The use of generating functions to characterize sequences is a common resource for transforming problems about sequences into problems involving functions formal power series. Following [6], Appell polynomials are characterized by a generating function of the form

$$
f(t) e^{x t}=\sum_{n=0}^{+\infty} p_{n}(x) \frac{t^{n}}{n !}
$$

where $f(t)$ is a real entire function.

Making use of the Cauchy product rule, the Taylor expansion of $f(t)$,

$$
f(t)=\sum_{n=0}^{+\infty} c_{n} \frac{t^{n}}{n !}, \quad f(0) \neq 0
$$

together with (4) allows to write Appell polynomials in the form

$$
p_{n}(x)=\sum_{k=0}^{n}\left(\begin{array}{l}
n \\
k
\end{array}\right) c_{n-k} x^{k}, \quad n=0,1, \ldots, \quad c_{0} \neq 0 .
$$

Remark 1. We observe that $c_{n}=p_{n}(0)$ and (5) is the exponential generating function of the number sequence $\left\{p_{n}(0)\right\}_{n \geq 0}$. 
Formula (4) shows that different polynomial sequences belonging to the Appell class correspond to different suitable choices of the function $f(t)$.

It is well known that Appell polynomials and corresponding number sequences have remarkable applications in several areas of mathematics (see, for instance, [5], [12]) but also in physics, chemistry and engineering (see, for instance, [13], [15]). This fact justifies the development of a matrix approach as an easy and comprehensible tool, also for non-mathematicians.

In [2] the authors have used the matrix $H$, of order $m+1$, (see (1)) which is nilpotent of degree $(m+1)$, i.e.

$$
H^{k}=0, \quad \forall k \geq m+1,
$$

in order to study some special types of polynomials of one real variable and to derive their properties.

This idea has been further developed in [23] into a unified matrix approach to Appell polynomials of one and several real variables. The general approach in the case of one real variable can briefly be described as follows.

Consider the vector of Appell polynomials

$$
\mathbf{p}(x)=\left[p_{0}(x) p_{1}(x) \cdots p_{m}(x)\right]^{T},
$$

and the vector of monomial powers

$$
\xi(x)=\left[\begin{array}{llll}
1 & x & \cdots & x^{m}
\end{array}\right]^{T} .
$$

By statement (2), $\mathbf{p}(x)$ satisfies the differential equation

$$
\frac{d}{d x} \mathbf{p}(x)=H \mathbf{p}(x)
$$

whose general solution is

$$
\mathbf{p}(x)=e^{x H} \mathbf{p}(0) .
$$

Remark 2. We recall that, $e^{x H}=P(x)$, where $P(x)$ is the $(m+1) \times(m+1)$ generalized Pascal matrix:

$$
\left[\begin{array}{cccccc}
1 & 0 & \ldots & \ldots & \ldots & \ldots \\
x & 1 & 0 & \ldots & \ldots & \ldots \\
x^{2} & 2 x & 1 & 0 & \ldots & \ldots \\
\vdots & \vdots & \vdots & \ddots & \vdots & \vdots \\
\left(\begin{array}{c}
m \\
0
\end{array}\right) x^{m} & \left(\begin{array}{c}
m \\
1
\end{array}\right) x^{m-1} & \left(\begin{array}{c}
m \\
2
\end{array}\right) x^{m-2} & \ldots & \ldots & \left(\begin{array}{c}
m \\
m
\end{array}\right)
\end{array}\right]
$$

since $P(1)=P$ is the ordinary lower triangular Pascal matrix. It is easy to verify that $P(-1)=P^{-1}$ is the inverse of $P($ see $[7])$. 
The relation (6) permits to represent (7) in the form

$$
\mathbf{p}(x)=M \xi(x),
$$

where $M$ is the $(m+1) \times(m+1)$ matrix defined by

$$
(M)_{i j}= \begin{cases}\left(\begin{array}{l}
i \\
j
\end{array}\right) c_{i-j}, & i \geq j \\
0, & \text { otherwise, } \quad i, j=0,1, \ldots, m\end{cases}
$$

Thus, the goal of achieving the matrix representation of the different types of Appell sequences implies to know $M$, the matrix that converts the vector $\xi(x)$ into a vector of Appell polynomials. It can be proved that such matrix $M$ coincides with the matrix

$$
f(H)=\sum_{n=0}^{m} c_{n} \frac{H^{n}}{n !}, \quad c_{0} \neq 0
$$

obtained as matrix polynomial from the corresponding Taylor polynomial of degree $(m+1)$ of $f(t)$ in $(4)$ (see [23]).

\section{Frobenius-Euler Polynomials and Special Matrices}

The general term of the Frobenius-Euler polynomial sequence $\left\{F_{n}(x ; \lambda)\right\}_{n \geq 0}$ is defined by the generating function

$$
\frac{1-\lambda}{e^{t}-\lambda} e^{x t}=\sum_{n=0}^{+\infty} F_{n}(x ; \lambda) \frac{t^{n}}{n !}
$$

where $\lambda \in \mathbb{C} \backslash\{1\}^{3}$.

This sequence is clearly an Appell sequence with

$$
f(t)=\frac{1-\lambda}{e^{t}-\lambda} .
$$

Special values of $x$ and $\lambda$ lead to the following examples.

(i) For $x=0, F_{n}(0 ; \lambda):=F_{n}(\lambda)$ are called Frobenius-Euler numbers (see [11]).

(ii) For $\lambda=-1, F_{n}(x ;-1)=E_{n}(x)$, which denotes the Euler polynomials.

(iii) For $\lambda=-1$ and $x=\frac{1}{2}, 2^{n} F_{n}\left(\frac{1}{2} ;-1\right)=E_{n}$, which denotes the Euler numbers.

Remark 3. Some authors (see [11]) call $F_{n}(0 ;-1)=E_{n}(0)$ Euler numbers. Here we refer to the common definition of Euler numbers, i.e., the numbers defined by the generating function

$$
\frac{2 e^{t}}{e^{2 t}+1}=\sum_{n=0}^{\infty} E_{n} \frac{t^{n}}{n !}
$$

\footnotetext{
$\overline{{ }^{3} \text { Carlitz ([8]) }}$ refers to these polynomials as Eulerian polynomials.
} 
which implies

$$
E_{n}=2^{n} E_{n}\left(\frac{1}{2}\right)
$$

(cf. [1]).

According to (6) it is obvious that

$$
F_{n}(x ; \lambda)=\sum_{k=0}^{n}\left(\begin{array}{l}
n \\
k
\end{array}\right) F_{n-k}(\lambda) x^{k}
$$

Likewise, from (13), using power series expansions and the Cauchy product, the following recurrence formula can be derived

$$
\sum_{k=0}^{n}\left(\begin{array}{l}
n \\
k
\end{array}\right) F_{k}(x ; \lambda)-\lambda F_{n}(x ; \lambda)=(1-\lambda) x^{n}, \quad n=0,1, \ldots
$$

The Frobenius-Euler polynomials up to $n=3$ are:

$$
\begin{aligned}
& F_{0}(x ; \lambda)=1 \\
& F_{1}(x ; \lambda)=x-\frac{1}{1-\lambda} \\
& F_{2}(x ; \lambda)=x^{2}-\frac{2}{1-\lambda} x+\frac{1+\lambda}{(1-\lambda)^{2}} \\
& F_{3}(x ; \lambda)=x^{3}-\frac{3}{1-\lambda} x^{2}+\frac{3(1+\lambda)}{(1-\lambda)^{2}} x-\frac{\lambda^{2}+4 \lambda+1}{(1-\lambda)^{3}} .
\end{aligned}
$$

In the very recent papers $[17,18]$ sequences $\left\{F_{n}^{(\alpha)}(x ; \lambda)\right\}_{n \geq 0}$ of FrobeniusEuler polynomials of order $\alpha, \alpha \in \mathbb{N}_{0}$, are considered. Those generalized sequences are defined by

$$
\left(\frac{1-\lambda}{e^{t}-\lambda}\right)^{\alpha} e^{x t}=\sum_{n=0}^{+\infty} F_{n}^{(\alpha)}(x ; \lambda) \frac{t^{n}}{n !},
$$

where $\lambda \in \mathbb{C} \backslash\{1\}$.

We can easily recognize that (17) includes the following special cases.

(i) When $\alpha=0$ the sequence $\left\{F_{n}^{(0)}(x ; \lambda)\right\}_{n \geq 0}$ corresponds to the monomials $\left\{x^{n}\right\}_{n \geq 0}$.

(ii) The case $\alpha=1$ corresponds to the Frobenius-Euler polynomials (13).

(iii) The case $\lambda=-1$ corresponds to the generalized Euler polynomials ([10])

(iv) The case $\alpha=2$ corresponds to the 2-iterated Euler polynomials, $E_{n}^{[2]}(x)$,

$$
\left(\frac{2}{e^{t}+1}\right)^{2}=\sum_{n=0}^{+\infty} E_{n}^{[2]}(x) \frac{t^{n}}{n !}
$$

introduced in [16]. 
According to (12) and (17), the corresponding matrix for the Frobenius-Euler polynomials of order $\alpha$ is

$$
\begin{aligned}
M_{\alpha} & =(1-\lambda)^{\alpha}\left(\left(e^{H}-\lambda I\right)^{\alpha}\right)^{-1} \\
& =(1-\lambda)^{\alpha}\left((P-\lambda I)^{\alpha}\right)^{-1} .
\end{aligned}
$$

Remark 4. When $\alpha=1, M \equiv M_{1}=(1-\lambda)(P-\lambda I)^{-1}$ is the corresponding matrix for the Frobenius-Euler polynomials, $F_{n}(x ; \lambda)$. Additionally, if $\lambda=-1$, then $M=2(P+I)^{-1}$ is the matrix referred in [2] for the Euler polynomials. For example, if $m=4$ one has

$$
M=\left[\begin{array}{ccccc}
1 & 0 & 0 & 0 & 0 \\
-\frac{1}{2} & 1 & 0 & 0 & 0 \\
0 & -1 & 1 & 0 & 0 \\
\frac{1}{4} & 0 & -\frac{3}{2} & 1 & 0 \\
0 & 1 & 0 & -2 & 1
\end{array}\right]
$$

From (10) and (18), the vector whose entries are Frobenius-Euler polynomials of order $\alpha$

$$
\mathbf{F}^{(\alpha)}(x ; \lambda)=\left[F_{0}^{(\alpha)}(x ; \lambda) F_{1}^{(\alpha)}(x ; \lambda) \cdots F_{m}^{(\alpha)}(x ; \lambda)\right]^{T},
$$

is readily obtained by

$$
\begin{aligned}
\mathbf{F}^{(\alpha)}(x ; \lambda) & =M_{\alpha} \xi(x) \\
& =(1-\lambda)^{\alpha}\left((P-\lambda I)^{\alpha}\right)^{-1} \xi(x) .
\end{aligned}
$$

Since (19),

$$
M_{\alpha}^{-1} \mathbf{F}^{(\alpha)}(x ; \lambda)=\xi(x)
$$

equivalent to

$$
(P-\lambda I)^{\alpha} \mathbf{F}^{(\alpha)}(x ; \lambda)=(1-\lambda)^{\alpha} \xi(x),
$$

which, for $\alpha=1$, represents (16) in matrix form.

Setting for the inverse matrix of $M_{\alpha}$

$$
M_{\alpha}^{-1}=\sum_{n=0}^{m} d_{n}^{(\alpha)} \frac{H^{n}}{n !}
$$

and taking into account (18), we have for the Frobenius-Euler polynomials of order $\alpha$

$$
d_{0}^{(\alpha)}=1, \quad d_{k}^{(\alpha)} \equiv \frac{1}{(1-\lambda)^{\alpha}}, \quad k=1, \ldots, m .
$$

From (20) and (21) it follows 


$$
\sum_{k=0}^{n}\left(\begin{array}{l}
n \\
k
\end{array}\right) d_{n-k}^{(\alpha)} F_{k}^{(\alpha)}(x ; \lambda)=x^{n} \quad n=0,1, \ldots
$$

which permits to derive the recurrence relation

$$
\left\{\begin{array}{l}
F_{0}^{(\alpha)}(x ; \lambda)=1 \\
F_{n}^{(\alpha)}(x ; \lambda)=\frac{1}{d_{0}^{(\alpha)}}\left(x^{n}-\sum_{k=0}^{n-1}\left(\begin{array}{l}
n \\
k
\end{array}\right) d_{n-k}^{(\alpha)} F_{k}^{(\alpha)}(x ; \lambda)\right), \quad n=1, \ldots, m .
\end{array}\right.
$$

\section{Some Identities of Frobenius-Euler Polynomials}

The approach presented in the previous section allows to derive several new identities involving Frobenius-Euler polynomials. Some of these identities are reported in [11] and [18] but not using the matrix representation of the polynomials.

Let us introduce the diagonal matrix

$$
D[-1]=\operatorname{diag}\left[1,-1,1, \ldots,(-1)^{m}\right] .
$$

Supposing $\alpha=1, \lambda \neq-1$ and $\lambda \neq 0$, from (19) we can derive the following:

$$
\begin{aligned}
\mathbf{F}\left(1-x ;-\frac{1}{\lambda}\right) & =\left(1+\frac{1}{\lambda}\right)\left(P+\frac{1}{\lambda} I\right)^{-1} \xi(1-x) \\
& =(1+\lambda)(\lambda P+I)^{-1} D[-1] P^{-1} \xi(x) \\
& =(1+\lambda)(D[-1](\lambda P+I))^{-1} P^{-1} \xi(x) .
\end{aligned}
$$

Further simplifications on the right side result in

$$
(1+\lambda) D[-1](P+\lambda I)^{-1} \xi(x)=D[-1] \mathbf{F}(x ;-\lambda) .
$$

Therefore, we obtain the following proposition.

Proposition 1 For $\lambda \neq-1$ and $\lambda \neq 0$, one has

$$
\mathbf{F}\left(1-x ;-\frac{1}{\lambda}\right)=D[-1] \mathbf{F}(x ;-\lambda) \text {. }
$$

Identity (22) is the matrix form of the symmetry property

$$
F_{n}\left(1-x ;-\frac{1}{\lambda}\right)=(-1)^{n} F_{n}(x ;-\lambda), \quad n=0,1, \ldots
$$

shown in [11] using a different method.

From (17) and (18) follows easily that

$$
\begin{aligned}
\mathbf{F}^{(\alpha+\beta)}(x+y ; \lambda) & =(1-\lambda)^{\alpha+\beta}\left((P-\lambda I)^{\alpha+\beta}\right)^{-1} \xi(x+y) \\
& =M_{\alpha} M_{\beta} \xi(x+y) .
\end{aligned}
$$


Since $\xi(x+y)=P(y) \xi(x)($ see $[2])$,

$$
\begin{aligned}
\mathbf{F}^{(\alpha+\beta)}(x+y ; \lambda) & =M_{\alpha} M_{\beta} P(y) \xi(x) \\
& =P(y) M_{\alpha} M_{\beta} \xi(x) \\
& =P(y) M_{\beta} \mathbf{F}^{(\alpha)}(x ; \lambda)
\end{aligned}
$$

( $M_{\alpha}, M_{\beta}, P(y)$ commute because they are functions of $H$.)

Therefore we obtain the following proposition.

Proposition 2 Let $\left\{F_{n}^{(\alpha)}(x ; \lambda)\right\}_{n \geq 0},\left\{F_{n}^{(\beta)}(x ; \lambda)\right\}_{n \geq 0}$ be two Frobenius-Euler polynomial sequences and $P(x)$ the generalized Pascal matrix. Then

$$
\mathbf{F}^{(\alpha+\beta)}(x+y ; \lambda)=P(y) M_{\beta} \mathbf{F}^{(\alpha)}(x ; \lambda) .
$$

Setting $\beta=0$ in (23) we obtain the following corollary.

Corollary 1 Let $\left\{F_{n}^{(\alpha)}(x ; \lambda)\right\}_{n \geq 0}$ be a Frobenius-Euler polynomial sequence and $P(x)$ the generalized Pascal matrix. Then

$$
\mathbf{F}^{(\alpha)}(x+y ; \lambda)=P(y) \mathbf{F}^{(\alpha)}(x ; \lambda) .
$$

The formula (24) is nothing else than the matrix representation of the identity

$$
F_{n}^{(\alpha)}(x+y ; \lambda)=\sum_{k=0}^{n}\left(\begin{array}{l}
n \\
k
\end{array}\right) F_{k}^{(\alpha)}(x ; \lambda) y^{n-k}, \quad n=0,1, \ldots
$$

reported in [18]. Such identity is an immediate consequence of the result which states that all Appell sequences $\left\{p_{n}(x)\right\}_{n \geq 0}$ fulfill a binomial theorem of the form

$$
p_{n}(x+y)=\sum_{k=0}^{n}\left(\begin{array}{l}
n \\
k
\end{array}\right) p_{k}(x) y^{n-k}, \quad n=0,1, \ldots
$$

(see [9]).

Furthermore, if $\lambda=-1$ then the identity (25) corresponds to the well known binomial expansion

$$
E_{n}^{(\alpha)}(x+y)=\sum_{k=0}^{n}\left(\begin{array}{l}
n \\
k
\end{array}\right) E_{k}^{(\alpha)}(x) y^{n-k}, \quad n=0,1, \ldots
$$

involving generalized Euler polynomials (cf. [21]).

It is worth noting that, setting $y=1$ and $y=(c-1) x$ in (24) and using the fact that $P(1)=P$, one get

$$
\begin{gathered}
\mathbf{F}^{(\alpha)}(x+1 ; \lambda)=P \mathbf{F}^{(\alpha)}(x ; \lambda), \\
\mathbf{F}^{(\alpha)}(c x ; \lambda)=P((c-1) x) \mathbf{F}^{(\alpha)}(x ; \lambda),
\end{gathered}
$$


respectively.

Identity (27) corresponds to a multiplication theorem of the form

$$
F_{n}^{(\alpha)}(c x ; \lambda)=\sum_{k=0}^{n}\left(\begin{array}{l}
n \\
k
\end{array}\right) F_{k}^{(\alpha)}(x ; \lambda)(c-1)^{n-k} x^{n-k}, \quad n=0,1, \ldots
$$

Moreover, from (18) and (26), one get successively

$$
\begin{aligned}
\mathbf{F}^{(\alpha)}(x+1 ; \lambda)-\lambda \mathbf{F}^{(\alpha)}(x ; \lambda) & =(P-\lambda I) \mathbf{F}^{(\alpha)}(x ; \lambda) \\
& =(1-\lambda)^{\alpha}(P-\lambda I)\left((P-\lambda I)^{\alpha}\right)^{-1} \xi(x) \\
& =(1-\lambda)^{\alpha}(P-\lambda I)\left((P-\lambda I)^{\alpha-1}(P-\lambda I)\right)^{-1} \xi(x) \\
& =(1-\lambda)(1-\lambda)^{\alpha-1}(P-\lambda I)(P-\lambda I)^{-1}\left((P-\lambda I)^{\alpha-1}\right)^{-1} \xi(x) \\
& =(1-\lambda) \mathbf{F}^{(\alpha-1)}(x ; \lambda) .
\end{aligned}
$$

Therefore, we have established the following result.

Proposition 3 For $\alpha \in \mathbb{N}$, the following recurrence relation is true

$$
\mathbf{F}^{(\alpha-1)}(x ; \lambda)=\frac{1}{(1-\lambda)}\left\{\mathbf{F}^{(\alpha)}(x+1 ; \lambda)-\lambda \mathbf{F}^{(\alpha)}(x ; \lambda)\right\} .
$$

Considering $\alpha=1$ in (28) and recalling that $\mathbf{F}^{(0)}(x ; \lambda)=\xi(x)$, we have

$$
\mathbf{F}(x+1 ; \lambda)-\lambda \mathbf{F}(x ; \lambda)=(1-\lambda) \xi(x) .
$$

Formula (28) in Proposition 3 allows to prove another recurrence relation.

Proposition 4 For $\alpha \in \mathbb{N}$, the following recurrence relation is true

$$
\mathbf{F}^{(\alpha-1)}(x ; \lambda)=\frac{1}{1-\lambda}(P-\lambda I) \mathbf{F}^{(\alpha)}(x ; \lambda) .
$$

Proof. From (24), with $x=1$ and $y \rightarrow x$, yields

$$
\begin{aligned}
\mathbf{F}^{(\alpha)}(x+1 ; \lambda) & =P(x) \mathbf{F}^{(\alpha)}(1 ; \lambda) \\
& =P(x) M_{\alpha} \xi(1) \\
& =P(x) M_{\alpha} P \xi(0),
\end{aligned}
$$

and because $P(x), M_{\alpha}, P$ commute,

$$
\begin{aligned}
\mathbf{F}^{(\alpha)}(x+1 ; \lambda) & =M_{\alpha} P P(x) \xi(0) \\
& =M_{\alpha} P \xi(x) \\
& =P \mathbf{F}^{(\alpha)}(x ; \lambda) .
\end{aligned}
$$

Then, together with (28), we get

$$
(1-\lambda) \mathbf{F}^{(\alpha-1)}(x ; \lambda)=(P-\lambda I) \mathbf{F}^{(\alpha)}(x ; \lambda),
$$

which completes the proof. 
The case $\lambda=-1$ leads to the relation between vectors of generalized Euler polynomials

$$
\mathbf{E}^{(\alpha-1)}(x ;-1)=\frac{1}{2}(P+I) \mathbf{E}^{(\alpha)}(x ;-1),
$$

which corresponds to the well known idendity

$$
E_{n}^{(\alpha-1)}(x)=\frac{1}{2}\left[E_{n}^{(\alpha)}(x)+\sum_{k=0}^{n}\left(\begin{array}{l}
n \\
k
\end{array}\right) E_{k}^{(\alpha)}(x)\right], \quad n=0,1, \ldots,
$$

referred in [10].

When $\alpha=1$, from (29), follows

$$
\xi(x)=\frac{1}{1-\lambda}(P-\lambda I) \mathbf{F}(x ; \lambda)
$$

which corresponds, when $\lambda=-1$, to

$$
x^{n}=\frac{1}{2}\left[E_{n}(x)+\sum_{k=0}^{n}\left(\begin{array}{l}
n \\
k
\end{array}\right) E_{k}(x)\right], \quad n=0,1, \ldots,
$$

referred in [21].

The above approach allows the matrix treatment of some extensions of FrobeniusEuler polynomials. For instance, of the generalized Apostol-Euler polynomials of order $\alpha, \mathfrak{E}_{n}^{(\alpha)}(x ; \lambda)$, defined by the generating function

$$
\left(\frac{2}{\lambda e^{t}+1}\right)^{\alpha} e^{x t}=\sum_{n=0}^{+\infty} \mathfrak{E}_{n}^{(\alpha)}(x ; \lambda) \frac{t^{n}}{n !}
$$

(see $[10])$.

In fact, considering the vector of generalized Apostol-Euler polynomials of order $\alpha$,

$$
\mathfrak{E}^{(\alpha)}(x ; \lambda)=\left[\mathfrak{E}_{0}^{(\alpha)}(x ; \lambda) \mathfrak{E}_{1}^{(\alpha)}(x ; \lambda) \cdots \mathfrak{E}_{m}^{(\alpha)}(x ; \lambda)\right]^{T},
$$

we recognize their relationship with the Frobenius-Euler polynomials in the following form:

Proposition 5 For $\alpha \in \mathbb{N}_{0}, \lambda \neq 0$ and $\lambda \neq-1$, Apostol-Euler polynomials $\mathfrak{E}_{n}^{(\alpha)}(x ; \lambda)$ and Frobenius-Euler polynomials $\mathbf{F}^{(\alpha)}\left(x ;-\frac{1}{\lambda}\right)$ of degree $\alpha$ satisfy

$$
\mathfrak{E}^{(\alpha)}(x ; \lambda)=\left(\frac{2}{1+\lambda}\right)^{\alpha} \mathbf{F}^{(\alpha)}\left(x ;-\frac{1}{\lambda}\right) .
$$

Proof. Taking into account that

$$
\mathbf{F}^{(\alpha)}\left(x ;-\frac{1}{\lambda}\right)=(1+\lambda)^{\alpha}\left((\lambda P+I)^{\alpha}\right)^{-1} \xi(x)
$$

and

$$
\mathfrak{E}^{(\alpha)}(x ; \lambda)=2^{\alpha}\left((\lambda P+I)^{\alpha}\right)^{-1} \xi(x),
$$

we get immediately the result. 


\section{Conclusion}

The approach here exposed permits to obtain a matrix representation of FrobeniusEuler polynomials, not used so far and, - without being exhaustive - to prove in a straightforward manner some of their main properties. Moreover, due to the matrix representation, all results can be generalized in order to obtain also Frobenius-Euler polynomials in several variables. The main tool in this direction would be a block version of the matrix $H$ as used in [20], [22, 23].

\section{Acknowledgements}

The authors would like to thank the referees for their valuable comments.

\section{References}

1. Abramowitz, M., Stegun, I. A.: Handbook of Mathematical Functions with Formulas, Graphs, and Mathematical Tables. Dover Publications, New York (1972)

2. Aceto, L., Trigiante, D.: The Matrices of Pascal and Other Greats. Amer. Math. Monthly 108 (3), 232-245 (2001)

3. Appell, P., Sur une classe de polynômes. Ann. Sci. École Norm. Supér. 9, 119-144 (1880)

4. Araci, S., Acikgoz, M.: A note on the Frobenius-Euler numbers and polynomials associated with Bernstein polynomials. Adv. Stud. Contemp. Math. 22(3), 399-406 (2012)

5. Avram, F., Taqqu, M. S.: Noncentral Limit Theorems and Appell Polynomial. Ann. Probab. 15(2), 767-775 (1987)

6. Boas, R. P., Buck, R. C.: Polynomial expansions of analytic functions. Springer, Berlin (1964)

7. Call, G. S., Velleman, J.: Pascal's Matrices. Amer. Math. Monthly 100, 372-376 (1993)

8. Carlitz, L.: The product of two eulerian polynomials. Math. Magazine. 36 (1), 37-41 (1963)

9. Carlson, B. C.: Polynomials Satisfying a Binomial Theorem. J. Math. Anal. Appl. 32, 543-558 (1970)

10. Chen, S., Cai, Y., Luo, Q-M: An extension of generalized Apostol-Euler polynomials. Adv. Difference Equ. 61, (2013). doi:10.1186/1687-1847-2013-61

11. Choi, J., Kim, D. S., Kim, T., Kim, Y. H.: A Note on Some Identities of Frobenius-Euler Numbers and Polynomials. Int. J. Math. Math. Sci. (2012). doi:10.115/2012/861797

12. Costabile, F. A., Longo, E.: The Appell interpolation problem. J. Comp. Appl. Math. 236, 1024-1032 (2011)

13. Fairlie, D. B., Veselov, A. P.: Faulhaber and Bernoulli polynomials and solitons. Physica D 152-153, 47-50 (2000)

14. Frobenius, G.: Uber die Bernoulli'schen Zahlen und die Euler'schen Polynome. Sitzungsberichte der Preussischen Akademie der Wissenschaften, 809-847 (1910)

15. Grosset, M. P., Veselov, A. P.: Elliptic Faulhaber polynomials and Lamé densities of states. Int. Math. Res. Not., Article ID 62120, 31 pp. (2006). doi: $10.1155 / \mathrm{IMRN} / 2006 / 62120$ 
16. Khan, S., Raza, N.: 2-iterated Appell polynomials and related numbers. Appl. Math. Comput. 219, 9469-9483 (2013)

17. Kim, D. S., Kim, T.: Some new identities of Frobenius-Euler numbers and polynomials. J. Inequal. Appl. 307, (2012). doi: 10.1186/1029-242X-2012-307

18. Kim, D. S., Kim, T., Lee, S-H, Rim, S-H: A note on the higher-order FrobeniusEuler polynomials and Sheffer sequences. Adv. Difference Equ. 41, (2013). doi: 10.1186/1687-1847-2013-41

19. Kurt, B., Simsek, Y.: On the generalized Apostol-type Frobenius-Euler polynomials. Adv. Difference Equ. 2013, 1 (2013). doi: 10.1186/1687-1847-2013-1

20. Malonek, H., Tomaz, G.: Laguerre polynomials in several hypercomplex variables and their matrix representation. In: Murgante, B., Gervasi, O., Iglesias, A., Taniar, D., Apduhan, B. (eds.) Computational Science and Its Applications-ICCSA 2011, LNCS, vol. 6784, pp. 261-270. Springer-Verlag Berlin, Heidelberg (2011)

21. Srivastava, H. M., Pintér, Á.: Remarks on Some Relationships Between the Bernoulli and Euler Polynomials. Appl. Math. Lett. 17, 375-380 (2004)

22. Tomaz, G., Malonek, H. R.: Special Block Matrices and Multivariate Polynomials. In: Simos, T.E., Psihoyios, G., Tsitouras, Ch. (eds.) Numerical Analysis and Applied Mathematics (ICNAAM 2010). AIP Conference Proceedings, vol.1281, pp. 1515-1518. Melville, New York (2010)

23. Tomaz, G.: Polinómios de Appell multidimensionais e sua representação matricial. PhD-Thesis (in Portuguese), University of Aveiro (2012) 